\title{
A case of mucinous cystadenocarcinoma of the pancreas with spontaneous rupture diagnosed by endoscopic retrograde pancreatography
}

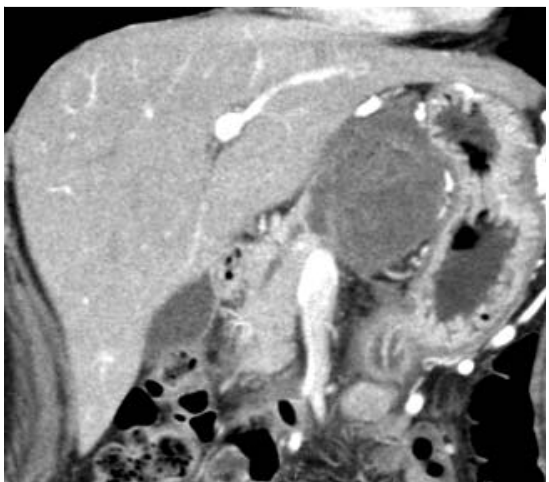

Fig. 1 Computed tomography shows the presence of a low-density mass in the pancreatic body that communicates with a dilated main pancreatic duct.

Mucinous cystic neoplasm (MCN) of the pancreas is characterized by a multilocular cystic lesion lined by mucin-producing columnar epithelium and an ovarian-type stroma [1]. MCNs are often found incidentally and may occasionally cause symptoms. Rupture of a pancreatic MCN is a rare complication, and only a few cases have been reported to date [2-5]. Many of these cases occurred during pregnancy $[2,3,5]$. This report is the first to describe a case of ruptured MCN with an associated invasive carcinoma of the pancreas diagnosed by endoscopic retrograde pancreatography (ERP).

In a 69-year-old woman complaining of abdominal pain, contrast-enhanced computed tomography (CT) showed a lowdensity mass $60 \mathrm{~mm}$ in diameter that communicated with a dilated main pancreatic duct ( Fig. 1). Contrast-enhanced endoscopic ultrasound (EUS) revealed a mixedecho pattern in the mass and enhancement effects in hyperechoic areas ( $\bullet$ Fig. 2). The findings on CT, EUS, and magnetic resonance imaging (not shown) suggested that the tumor was a multilocular cystic lesion of the pancreas with solid components, but they were atypical, and a diagnosis could not be reached. ERP was then performed and showed leakage of contrast medium from the main pancreatic duct into the peritoneal cavity ( Fig.3). CT images obtained immediately after ERP showed pooling of contrast medium in
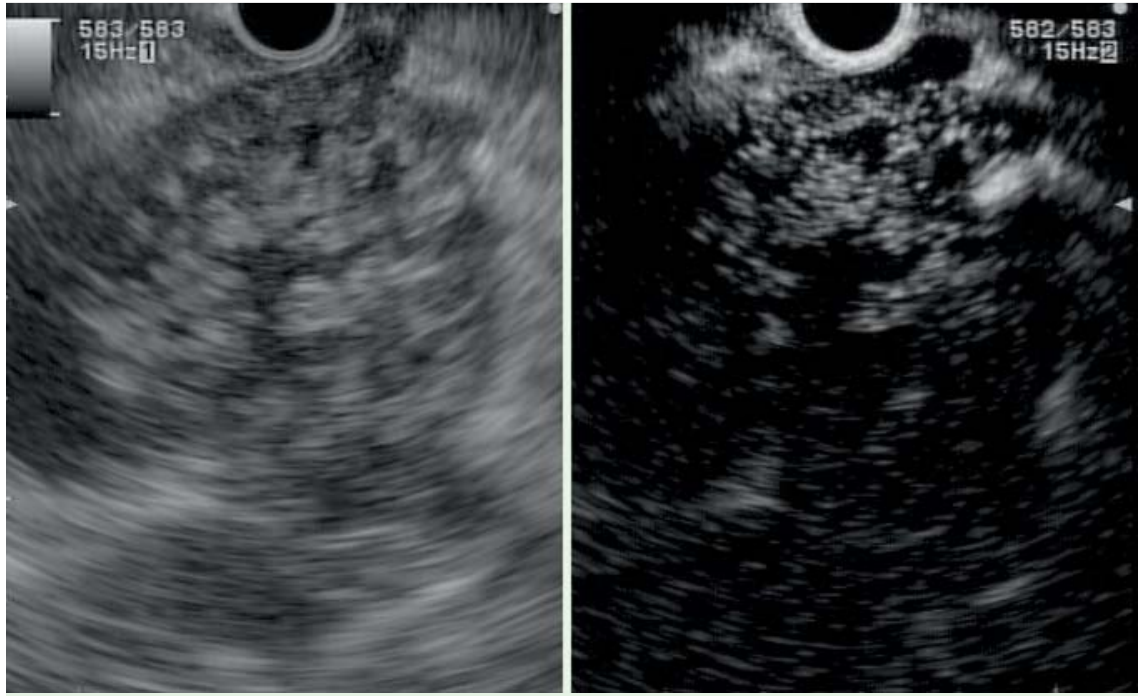

Fig.2 Contrast-enhanced endoscopic ultrasound shows a mixed-echo pattern in the mass and enhancement effects in hyperechoic areas.

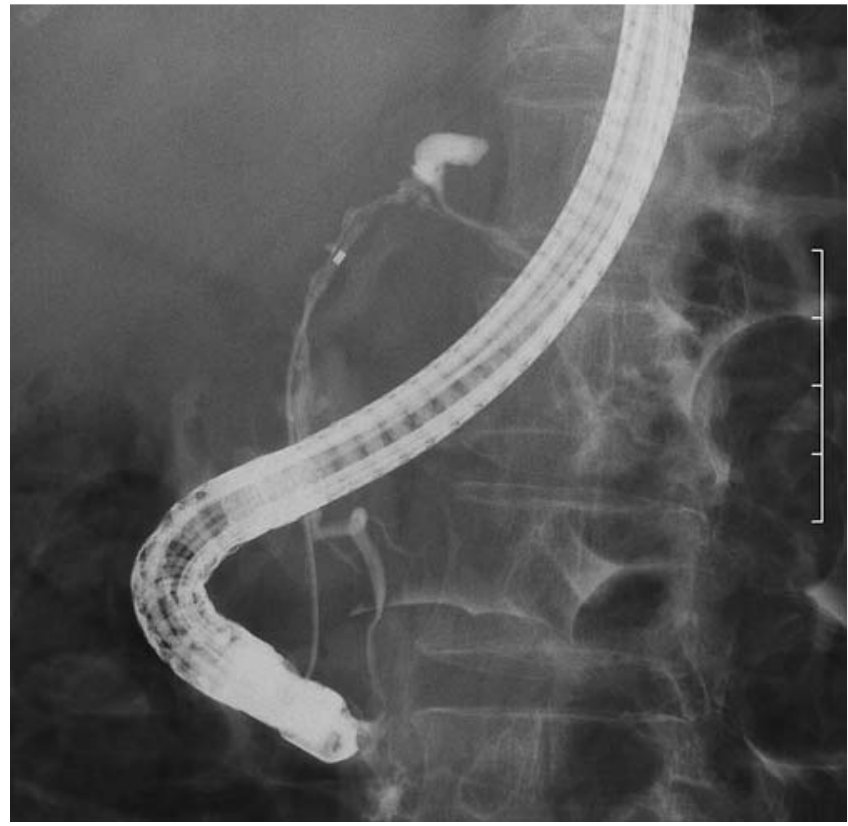

Fig. 3 Endoscopic retrograde pancreatography shows rupture of the tumor. Leaked contrast medium from the main pancreatic duct is pooling around the intestinal tract. Scale bar: $1 \mathrm{~cm}$.

the peritoneal cavity around the tumor ( Fig.4). A body-tail pancreatectomy with splenectomy was performed quasiemergently 5 days after ERP. The definitive pathological diagnosis was mucinous cystadenocarcinoma of the pancreas with foci of ovarian-like stroma ( Fig.5). After surgery, the patient underwent chemotherapy with 5-fluorouracil and has remained symptom-free, with no detectable tumor recurrence at present, 2 months after surgery.

To the best of our knowledge, this is the first report of MCN of the pancreas with spontaneous rupture diagnosed by ERP.

Endoscopy_UCTN_Code_CCL_1AZ_2AB

Competing interests: None 


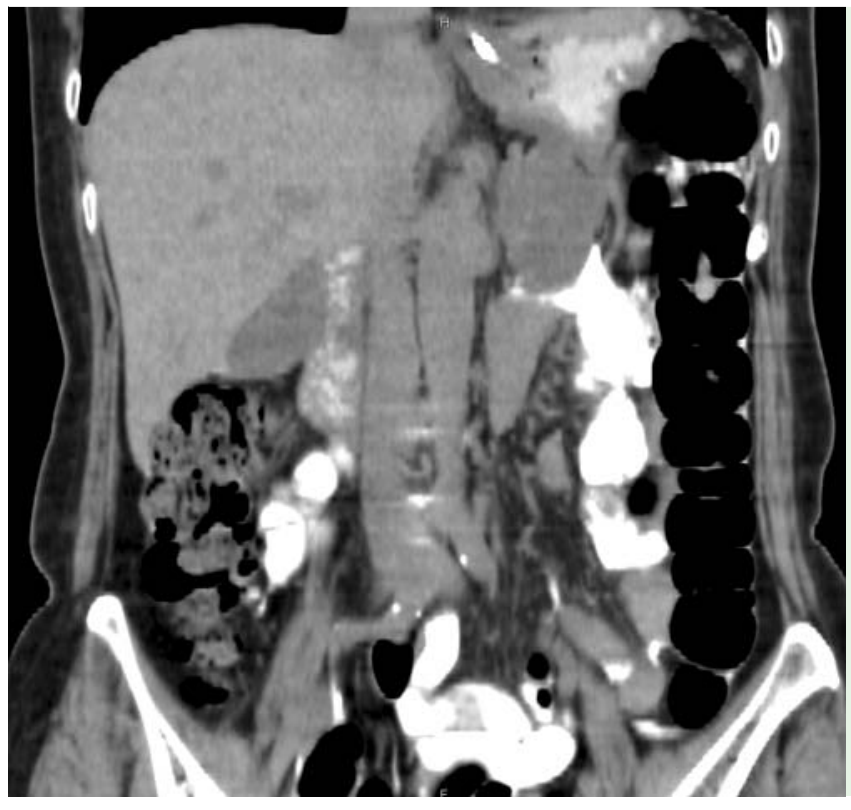

Fig. 4 Pooling of contrast medium is seen in the peritoneal cavity around the tumor.

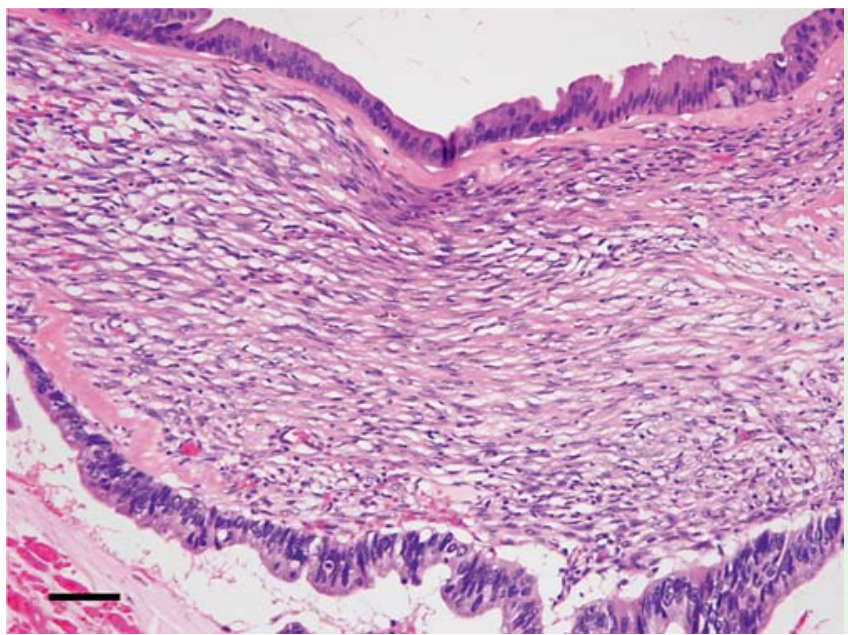

Fig. 5 Histological sections showed thickened mucinous epithelium and ovarian-type stroma (hematoxylin and eosin; magnification $\times 200$ ). Scale bar: $50 \mu \mathrm{m}$.

\section{A. Imoto ${ }^{1}$, D. Masuda', Y. Kurisu², S. Onda ${ }^{1}$, T. Ogura ${ }^{1}$, M. Takii ${ }^{1}$, E. Umegaki ${ }^{1}$, M. Hayashi ${ }^{3}$, K. Uchiyama ${ }^{3}$, K. Higuchi ${ }^{1}$}

${ }^{1}$ Second Department of Internal Medicine, Osaka Medical College, Takatsuki, Osaka, Japan

2 Department of Pathology, Osaka Medical College, Takatsuki, Osaka, Japan

3 Department of General and Gastroenterological Surgery, Osaka Medical College, Takatsuki, Osaka, Japan

\section{References}

1 Brugge WR, Lauwers GY, Sahani D et al. Cystic neoplasms of the pancreas. N Engl J Med 2004; 351: $1218-1226$

2 Naganuma S, Honda K, Noriki $S$ et al. Ruptured mucinous cystic neoplasm with an associated invasive carcinoma of pancreatic head in a pregnant woman: report of a case and review of literature. Pathol Int 2011; 61: $28-33$

3 Ozden S, Haliloglu B, Ilter E et al. An extremely rare cause of acute abdomen in pregnancy: ruptured pancreatic mucinous cystadenocarcinoma. Pancreas 2007; 34: 474-476

4 Bergenfeldt M, Poulsen IM, Hendel HW et al. Pancreatic ascites due to rupture of a mucinous cystic neoplasm. Acta Oncol 2008; 47: 978-981

5 Smithers BM, Welch C, Goodall P. Cystadenocarcinoma of the pancreas presenting in pregnancy. Br J Surg 1986; 73: 591

\section{Bibliography}

DOI http://dx.doi.org/

10.1055/s-0032-1325888

Endoscopy 2013; 45: E36-E37

(c) Georg Thieme Verlag KG

Stuttgart · New York

ISSN 0013-726X

\section{Corresponding author}

\section{A. Imoto, MD}

Second Department of Internal Medicine

Osaka Medical College

Takatsuki

Osaka 569-8686

Japan

Fax: +81-726-84-6423

akiraimoto@hotmail.co.jp 